\title{
Problems of Sampling and Inference in the Study of Fluctuating Dental Asymmetry
}

\author{
B. HOLLY SMITH, STANLEY M. GARN, AND PATRICIA E. COLE \\ Center for Human Growth and Development, The University of Michigan, \\ Ann Arbor, Michigan, 48109
}

\author{
KEY WORDS Fluctuating dental asymmetry, Sample size, Crown size
}

\begin{abstract}
Randomly distributed or "fluctuating" dental asymmetry has been accorded evolutionary meaning and interpreted as a result of environmental stress. However, except for congenital malformation syndromes, the determinants of human crown size asymmetry are still equivocal. Both a computer simulated sampling experiment using a combined sample size of $N=3000$, and the requirements of adequate statistical power show that sample sizes of several hundred are needed to detect population differences in dental asymmetry. Using the largest available sample of children with defined prenatal stresses, we are unable to find systematic increases in crown size asymmetry. Given sampling limitations and the current inability to link increased human dental asymmetry to defined prenatal stresses, we suggest that fluctuating dental asymmetry is not yet established as a useful and reliable measure of general stress in human populations.
\end{abstract}

More than 25 publications dealing with "fluctuating dental asymmetry" have appeared since Van Valen applied this concept to the dentition in 1962. While the majority of authors have regarded fluctuating dental asymmetry as a measure of the ability of an organism to buffer itself against developmental or genetic "stress," specific treatments of this thesis have varied considerably. Some authors have employed dental asymmetry as an indication of environmental stress in archaeological populations (Doyle and Johnston, 1977; Perzigian, 1977). A number of studies have attempted to test the "stress" hypothesis, using comparisons of archaeological collections, recent humans or experimental animals, with both positive and negative results (Niswander and Chung, 1965; Bailit et al., 1970; Siegel and Smookler, 1973; Siegel and Doyle, 1975; Siegel et al., 1977; DiBennardo and Bailit, 1978; Sciulli et al., 1979; Black, 1980). Other authors have concentrated on the hypothesis that there is a genetic basis for the capacity to buffer stress and it is the genetic factor and the environment that together influence levels of dental asymmetry (Van Valen. 1962; Bader, 1965; Bailit, 1966; Staley and Green, 1974; Potter and Nance, 1976; Corruccini and Potter, 1981). It has also been argued that fluctuating dental asymmetry reflects the operation of natural selection on tooth size (Suarez, 1974; Wolpoff, 1975).

In spite of these many studies, some descriptive and others experimental, using casts of living people, archaeological material, and experimental animals, several points deserve particular attention. The first is that estimates of dental asymmetry may differ depending on which statistic is used. Second, sampling effects may swamp existing differences in leftright crown size asymmetry. Dental asymmetry in human populations typically averages little more than $0.1 \mathrm{~mm}$, making most population differences difficult to establish. Only in Trisomy $\mathrm{G}+$ and cleft palate syndromes has fluctuating dental asymmetry consistently been shown to rise above the level of developmental "noise" (Adams and Niswander, 1967; Garn et al., 1970; Barden, 1980; Sofaer, 1979; Wilcox, 1981).

In view of these problems we have given attention to a number of methodological and statistical considerations that bear upon the study of fluctuating dental asymmetry. These include the best way to express crown size asymmetry, whether meaningful differences in fluctuating asymmetry can be demonstrated in

Received July 17, 1981; accepted December 22, 1981. 
samples of reasonable size, and whether certain prenatal stresses result in increased crown size asymmetry.

\section{EXPRESSING ASYMMETRY}

There are several ways to express left-right dental asymmetry. The mean of left-right differences in tooth size can be used to demonstrate "sidedness," but the mean difference will be zero even in the presence of considerable asymmetry if it is random with respect to side. For this reason, "fluctuating" or random asymmetry is commonly described by the simple correlation coefficient $\left(r_{\mid r}\right)$ or as the standard deviation of the difference of right and left side measurements $\left(s_{d}\right)$. The latter is termed root mean square (RMS) asymmetry.

RMS asymmetry has the advantage of being directly interpretable in the original units of measurement. For example, a RMS tooth size asymmetry value of 0.2 indicates that asymmetry falls between -0.2 and $+0.2 \mathrm{~mm}$ for $67 \%$ of the sample. However, RMS asymmetry itself is a function of crown size - an important problem in comparisons of different human populations. This factor alone might account for high levels of asymmetry in Neanderthals or American Indians in comparison with modern white populations (e.g., Doyle and Johnston, 1977).

The left-right correlation also has its problems, stemming from the distribution of values and the effect of measurement error when correlations approach 1.0, but it does take population differences in tooth size into account. The two measures $\left(r_{l r}\right.$ ands $\left.s_{d}\right)$ correspond perfectly only if the correlation is expressed as the coefficient of alienation $(1-r)$ and if RMS asymmetry is corrected for crown size variance by expressing it as left-right intrapair variance, i.e., $\operatorname{var}(R-L) / \operatorname{var}(R)+\operatorname{var}(L)$ (which we have confirmed by empirical tests). The appropriate measure of asymmetry to be used then depends on the nature of the hypothesis under test.

\section{SAMPLING CONSIDERA'TIONS}

The problem of sufficient sample size for the study of fluctuating dental asymmetry can be resolved empirically or mathematically.

To construct an empirical test we assembled a uniquely large sample of individuals from three different study samples: 656 from the Fels Research Institute (see Garn et al., 1966), 208 from The University of Michigan Longitudinal Study (see Moyers et al., 1976), and 2240 from the NINCDS National Collabora- tive Perinatal Project (NCPP) collected by Dr. Richard H. Osborne (see Garn et al., 1979). In all three data bases, mesiodistal and buccolingual crown dimensions were measured on casts.

Having first demonstrated that the component samples did not differ in crown size asymmetry tooth by tooth, the three were combined. For the purposes of the sampling experiment, the 3104 subjects can be regarded as a single population, although artificially constructed. This "population" included as many as 1322 observations for specific pairs of teeth. We drew eleven independent random samples of size $15,25,50,75,100,225,400,625,750,900$, and 1000 from the total for each tooth. These subsamples were examined for differences in estimates of asymmetry as a function of sample size.

Figure 1 summarizes the results for the 14 permanent teeth (I1-M2) using RMS asymmetry as the computed measure of asymmetry. Sample size (on the abscissa) is plotted against the corresponding estimate of RMS asymmetry (ordinate). Despite differences in RMS asymmetry for individual teeth, the results are instructive. Both the highest and lowest values of RMS asymmetry appear with the smallest sample sizes, as would be expected. The range of estimates of $\sigma_{\mathrm{d}}$ decreases substantially with very large Ns; the final range of values obtained with $\mathrm{N}=1000$ is half that for samples of $N<50$. As shown, estimates of $\sigma_{\mathrm{d}}$ do not appear to stabilize until sample sizes exceed 600 .

As an additional demonstration, the experiment was repeated with a total of 35 random samples of size $15,25,50,75,100,200$, and 300 for maxillary M2. Figure 2 shows that the resulting trend is the same whether RMS asymmetry or the left-right correlation is used as the measure of crown size asymmetry. The range of estimates of RMS asymmetry obtained when $N=300$ is $90 \%$ less than the range of values when $N<25$. Similarly, for the leftright correlation the range of computed $r s$ decreases by $85 \%$ for an $N$ of 300 compared with that observed at $\mathrm{N}<25$.

The conclusions to be drawn from this sampling experiment bear on the study of "stress" in archaeological human populations where small samples are the rule. A comparison of published asymmetry values with expected sampling limits strongly suggests that the use of small samples has influenced previous studies. Left-right crown size correlations were available for Indian Knoll, the 


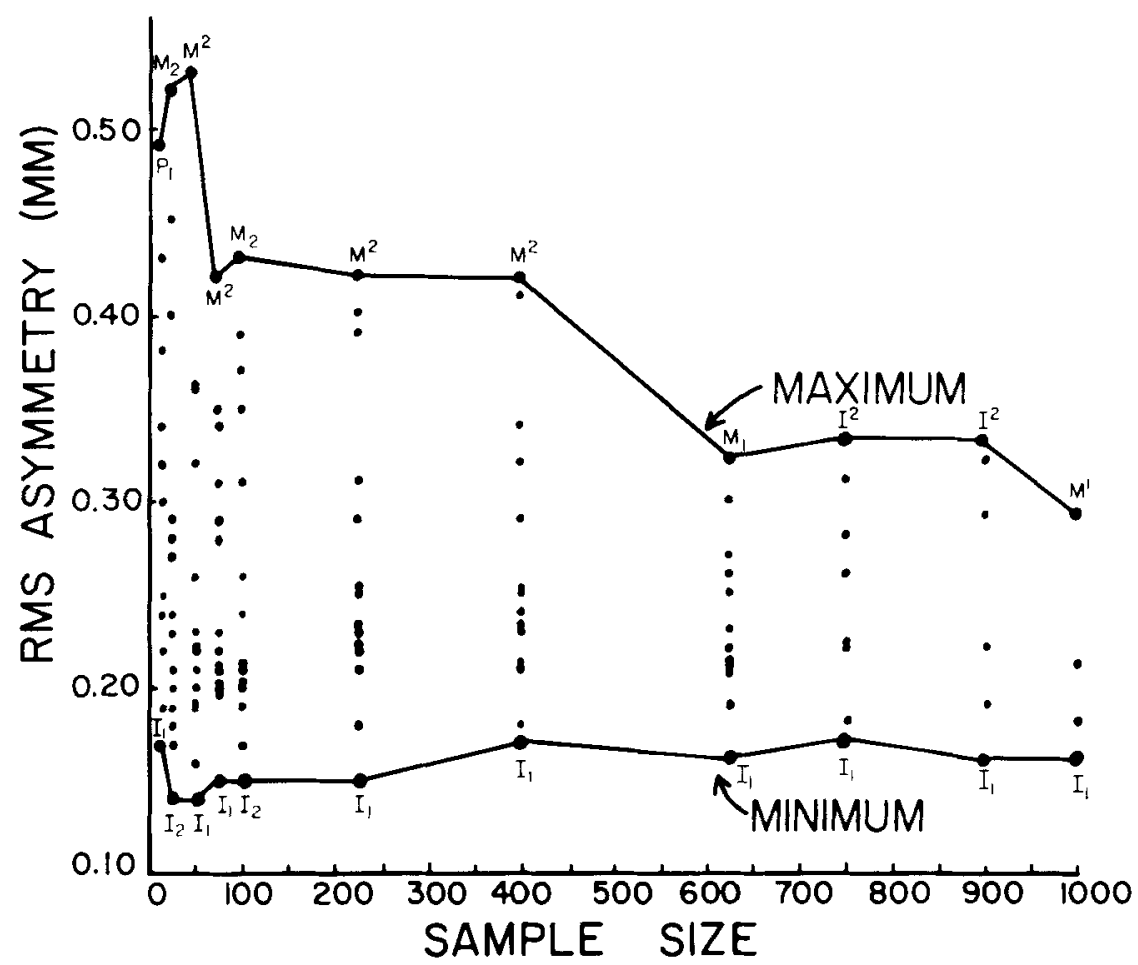

Fig. 1. The relationship between sample size (abscissa) and RMS crown size asymmetry (ordinate) using computer generated independent random subsamples from a single "population" of more than 3000 individuals. As shown by the actual values for each tooth and by the solid lines depicting minimum and maximum values for each sample size, the lowest and highest estimates of RMS crown size asymmetry are systematically associated with samples below 100 Beyond an N of 600 the range of RMS asymmetry values effectively stabilizes, indicating that rather large samples are needed in studies of crown size asymmetry.

The problem of appropriate sample sizes can also be resolved mathematically. For an example, we turn again to RMS asymmetry and the sample size necessary for a powerful test of the hypothesis that one population has higher dental asymmetry than another.

\section{STATISTICAL POWER}

The power of a statistical test is the probability that the null hypothesis will be rejected when it is in fact false. In other words, if there is a real difference in the variance of crown size difference, power is the chance that an $F$ test will find a statistically significant difference. The inverse of power $(1-\beta)$ is the familiar probability of a Type II error $(\beta)$-the chance that the null hypothesis will be mistakenly accepted when there is in fact a real difference (Dixon and Massey, 1969). Statistical power is particularly important when an absolutely 


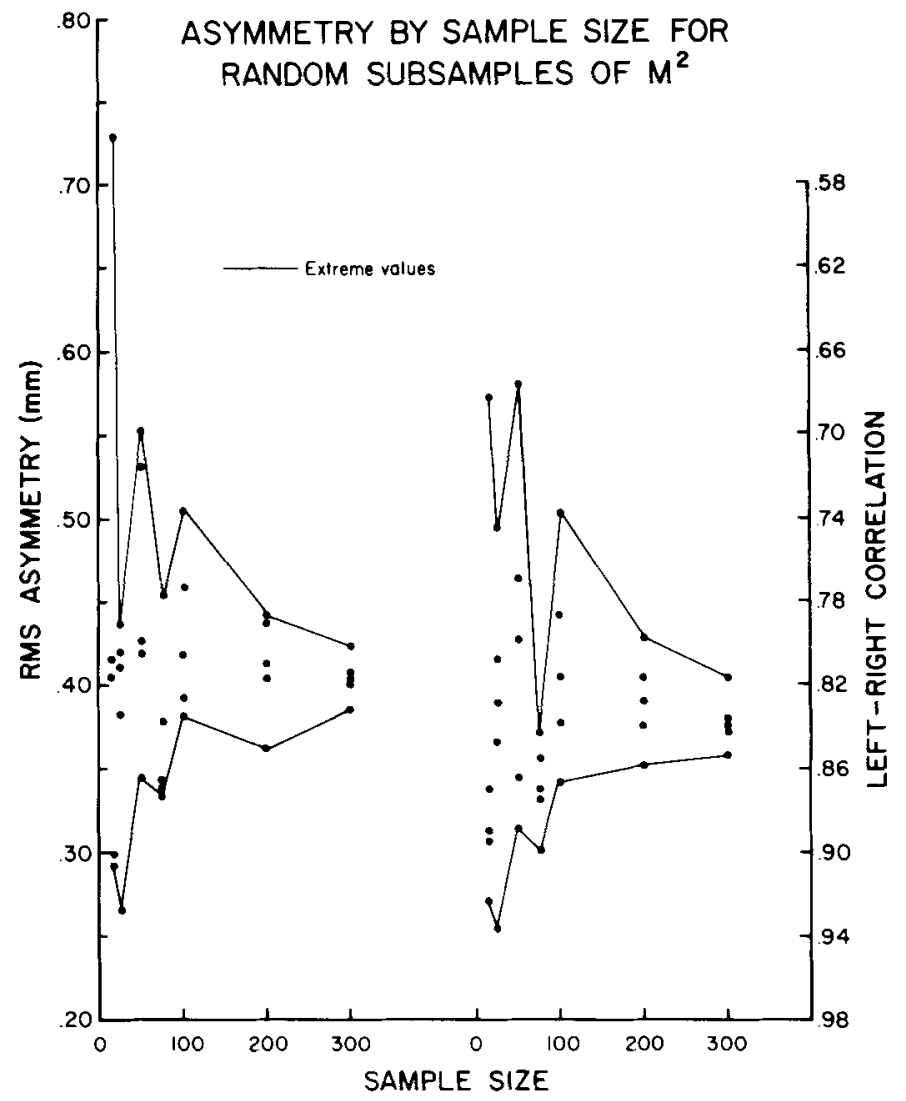

Fig. 2. Estimates of crown size asymmetry obtained from 35 computer generated, independent, random subsamples of maxillary M2 from a single population. Whether asymmetry is expressed as RMS (at left) or the left-right correlation (at right), trends are much the same: the range of variation of estimates is much greater for small samples ( $N$ $<25)$ than it is for large samples $(\mathrm{N}>200)$. small difference exists and tests are conducted with small sample sizes, as with crown size asymmetry. Continued negative results may be due to a lack of sufficient statistical power.

Using the $F$ test for equality of variances, the power of the test depends on the level of significance $(\alpha)$, the true ratio of the variances in the populations compared $(F)$, and the sample size used. To test the hypothesis that a "stressed" group has higher RMS crown size asymmetry $\left(\sigma_{d}\right)$ than a "normal" group, it is appropriate to compute a one-sided $F$ test at $\alpha=$ 0.05 . Given this situation, it is possible to construct a graph showing the relationship between sample size and the true ratio of the variances $\left(\sigma_{\mathrm{d}}^{2}\right.$ stressed $/ \sigma_{\mathrm{d}}{ }^{2}$ normal), for a particular level of statistical power. Figure 4 illustrates this for the simple case of balanced sample Ns and shows power curves for $25,50,75$, and $90 \%$. These percentages indicate the chance of success (rejection of the null hypothesis) given a single random sample when the "stressed" group in fact has higher RMS dental asymmetry than the "normal" group. Figure 4 shows that small samples $(\mathrm{N}<20$ ) have small chance of success unless the magnitude of the true variance ratio $(F)$ is very large. For a sample of $N_{1}=N_{2}=10$, a power of $90 \%$ would not be achieved unless the stressed population has a crown size asymmetry variance more than seven times that of normal. To detect a more reasonable difference, say, a twofold increase in crown size asymmetry variance at $75 \%$ power, samples of about 45 in each group would be needed. However, if the ratio of stressed to normal variance $(F)$ is as low as 1.5 , a sample of over 100 in each group would be necessary for a $75 \%$ chance of success. Indeed, at this moderate level of difference in variances, test with $N_{1}=N_{2}=60$ have a chance 


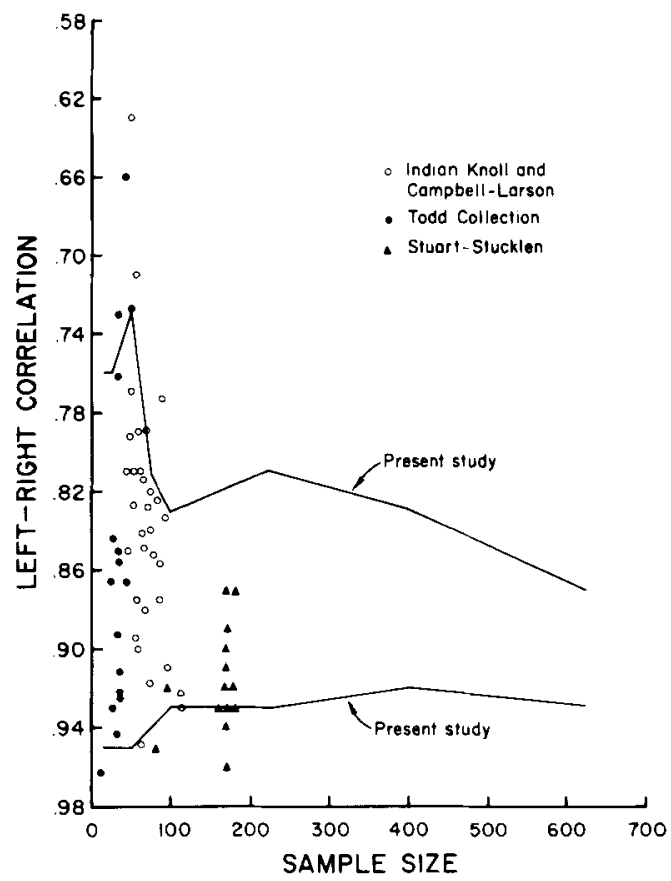

Fig. 3. Left-right correlation from Perzigian (1977) and Moorrees and Reed (1964) plotted against confidence limits for comparable sample sizes obtained by computer simulation (solid lines). Of the 62 published values, only 11 fall out side limits appropriate to sample size, suggesting that many of the apparent population differences in asymmetry may be the result of sampling effects. Values published by Bailit et al. (1970) were not used due to apparent differences in methods of calculation.

of success equal only to 0.5 , the chance of getting a head with a single toss of a coin.

\section{DEVELOPMENTAL STRESS}

Having shown the inherent risks of small samples in dental asymmetry problems, we next tested the hypothesis that fluctuating dental asymmetry is increased in the presence of stress using the largest currently available sample of individuals with defined prenatal stresses. The NINCDS Collaborative Perinatal Project (NCPP) data base contains dental measurements on both normal children and children of mothers with specified risk conditions (Garn et al., 1979). We investigated RMS dental asymmetry in NCPP children who had experienced defined prenatal stresses or were characterized by dimensional extremes at birth. The specific conditions considered include: (1) maternal diabetes, (2) maternal hypothyroidism, (3) maternal hypertension, (4) toxemias of pregnancy, (5) short gestation

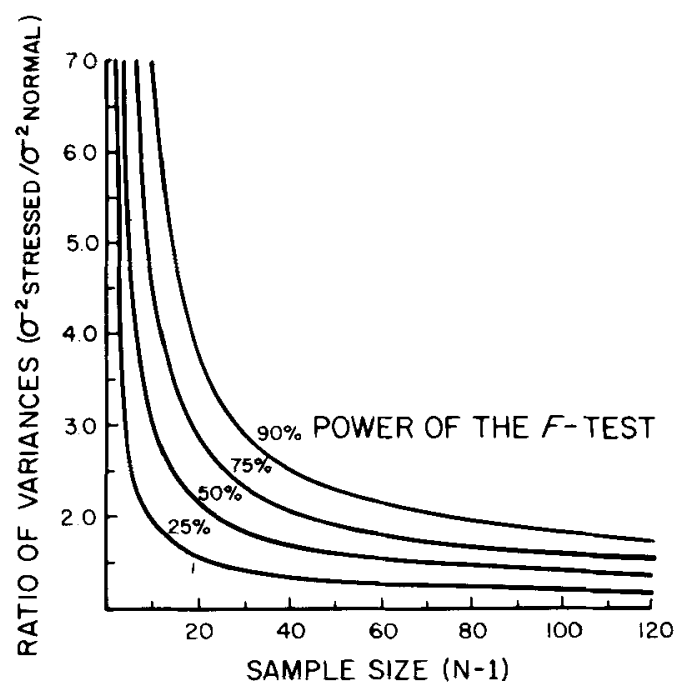

Fig. 4. The relationship between sample size $(\mathrm{N}-1$ in each group) and the true ratio of the variances $\left(F=\sigma_{\mathrm{d}}{ }^{2}\right.$ stressed $/ \sigma_{\mathrm{d}}{ }^{2}$ normal) at four levels of statistical power. Each power curve $(25,50,75$, and $90 \%)$ represents the probability of correctly rejecting the null hypothesis when there is in fact a real difference in crown size asymmetry variances. Note that if "stress" doubles variance $\left\langle\sigma_{\mathrm{d}}{ }^{2}\right.$ stressed $/ \sigma_{\mathrm{d}}{ }^{2}$ normal $=2$ ), a test with 10 in each group has a power of about $25 \%$, at $\mathrm{N}=25$ a power of $50 \%$, at $\mathrm{N}=40$ a power of $75 \%$, and at $\mathrm{N}=120$ a power of $90 \%$. As is evident, much larger samples are required if the effect of "stress" on variance of crown size differences is still less. Calculated from tables in Dixon and Massey (1969:472-485), the chart is valid for onesided $F$ tests at $\sigma=.05$ with balanced sample Ns.

length, (6) low birth weight, and (7) short birth length. In each case, specific maternal conditions have been compared with remaining subjects since the total sample was previously shown not to differ in RMS asymmetry from two separate normal series. In all, there were 184 comparisons of RMS asymmetry involving these prenatal and birth conditions to controls, performed separately for each tooth and race/sex group. Teeth investigated are those that develop prenatally and shortly after birth, which therefore might be affected by a prenatal insult, i.e., the deciduous teeth, and permanent incisors and M1. Rather than performing multiple $F$ tests on these small samples, we first asked if the stressed groups tended to show higher variance than controls, or if the direction of the difference was random with respect to group. For four maternal conditions (Table 1) there are 79 comparisons that met the minimum criterion of $N>25$ in each group. For all conditions combined, "abnormals" show greater asym- 
TABLE 1. Comparisons of RMS asymmetry between maternal conditions and control group

\begin{tabular}{|c|c|c|c|c|}
\hline Maternal condition & Teeth & $\begin{array}{l}\text { No. } \\
\text { comparisons }^{\mathrm{a}}\end{array}$ & $\begin{array}{l}\text { "Stressed" } \\
\text { RMS higher }\end{array}$ & $\begin{array}{l}\text { Sign } \\
\text { test }^{b}\end{array}$ \\
\hline Hypothyroid & $\mathrm{dm}^{2}$ & 3 & 2 & n.s. \\
\hline Diabetic & I1 12 & 5 & 5 & n.s. \\
\hline Hypertensive & II $\mathrm{I}_{2}$ & 3 & 0 & n.s. \\
\hline Toxemic & All $12^{\mathrm{c}}$ & 60 & 30 & n.s. \\
\hline All conditions, most avallable tooth & $\mathbf{I}_{1}$ & 5 & 3 & n.s. \\
\hline All conditions & AI: $12^{\mathrm{c}}$ & 79 & 40 & n.s. \\
\hline
\end{tabular}

a All sample sizes $>25$, with each tooth and racelsex group considered separately.

b.s. = not significant.

c All 12 teeth: dc, dm1, dm2. I1. [2, M1, both jaws.

TABLE 2. RMS asymmetry of neonates less than 20th percentile compared with those greater than 80th percentile for three gestational conditions

\begin{tabular}{|c|c|c|c|c|}
\hline Gestational condition & Teeth & $\begin{array}{c}\text { No. } \\
\text { comparisons }\end{array}$ & $\begin{array}{l}\text { "Stressed" } \\
\text { RMS higher }\end{array}$ & $\begin{array}{l}\text { Sign } \\
\text { test }\end{array}$ \\
\hline Low birth weight & $\mathrm{I} 1, \mathrm{I} 2, \mathrm{M} 1, \mathrm{dc}, \mathrm{dm}_{1}, \mathrm{dm} 2$ & $41^{\mathrm{a}}$ & 21 & n.s. \\
\hline Short birth length & All 12 teeth $^{c}$ & 30 & 14 & n.s. \\
\hline Short gestation & $\mathrm{I} 1, \mathrm{I} 2, \mathrm{M} 1, \mathrm{dc}, \mathrm{dm}_{1}, \mathrm{dm} 2$ & 34 & 20 & n.s. \\
\hline Total & All 12 teeth $^{\mathrm{c}}$ & 105 & 55 & n.s. \\
\hline
\end{tabular}

a All sample sizes $>25$, with each tooth and race/sex group considered separately.

$b_{\text {n.s. }}=$ not significant.

c All 12 teeth: dc, dm1, dm2, 11. I2, M1, both jaws.

metry in 40 of the 79 cases-almost exactly following chance expectancy. No tendency for increased asymmetry is observable in the stressed group.

For a rough approximation to the hypothesis that "poor nourishment" is an important contributor to increased dental asymmetry (Perzigian, 1977), we next chose to compare RMS asymmetry in "small" versus "large" neonates. Although diminished fetal growth may not resemble postnatal starvation, a positive result would strongly support a relationship between asymmetry and prenatal nutritional extremes. Boys and girls of low birth weight, initially short birth length, or short gestation $(<20$ th percentile) were compared to those with high birth weight, long birth length, and long gestastion (> 80th percentile). In 105 comparisons of $\mathrm{N}>25$ for each tooth and race/sex group, there are no particular tendencies for prematurely born $(<39$ weeks) or small infants ( $<47 \mathrm{cms},<2500 \mathrm{gms})$ to show increased crown size asymmetry (see Table 2).

In a further analysis we gave particular attention to children of toxemic mothers, the largest group for a single risk condition. Black and white, male and female children were each compared separately to the appropriate nontoxemic series from the NINCDS dental data base. Eight maxillary and eight mandibular teeth were compared with respect to RMS crown size asymmetry, with samples for the toxemic group of size 27-150, and size 47-283 for the control group. Table 3 presents the RMS values and $F$ ratios for all 60 comparisons. All $F$ values are calculated as $s_{d}{ }^{2}$ tox emic $/ \mathrm{s}_{\mathrm{d}}{ }^{2}$ control, so an $\mathrm{F}>1$ indicates support for the hypothesis that stress increases dental asymmetry. Toxemics show significantly greater asymmetry in 9 of 60 comparisons. If each test were independent we would expect at least three significant results from chance alone. The inclusion of the same individuals in many of the tests violates this assumption, although intercorrelations of asymmetry within individuals are reportedly low (Garn et al., 1966). Altogether, the number and seemingly random distribution of significant results for different teeth supports the case for no difference (the average of all $F$ values is 1.03). The only tooth measurement to show more than one significant difference for the four race/sex groupings is mesiodistal $\mathrm{dm}_{2}$ length, yet the buccolingual dimension of the same tooth is apparently unaffected. On the other hand, if we were to suggest that the toxemic group should show less asymmetry than controls, consistent with their slightly smaller tooth 


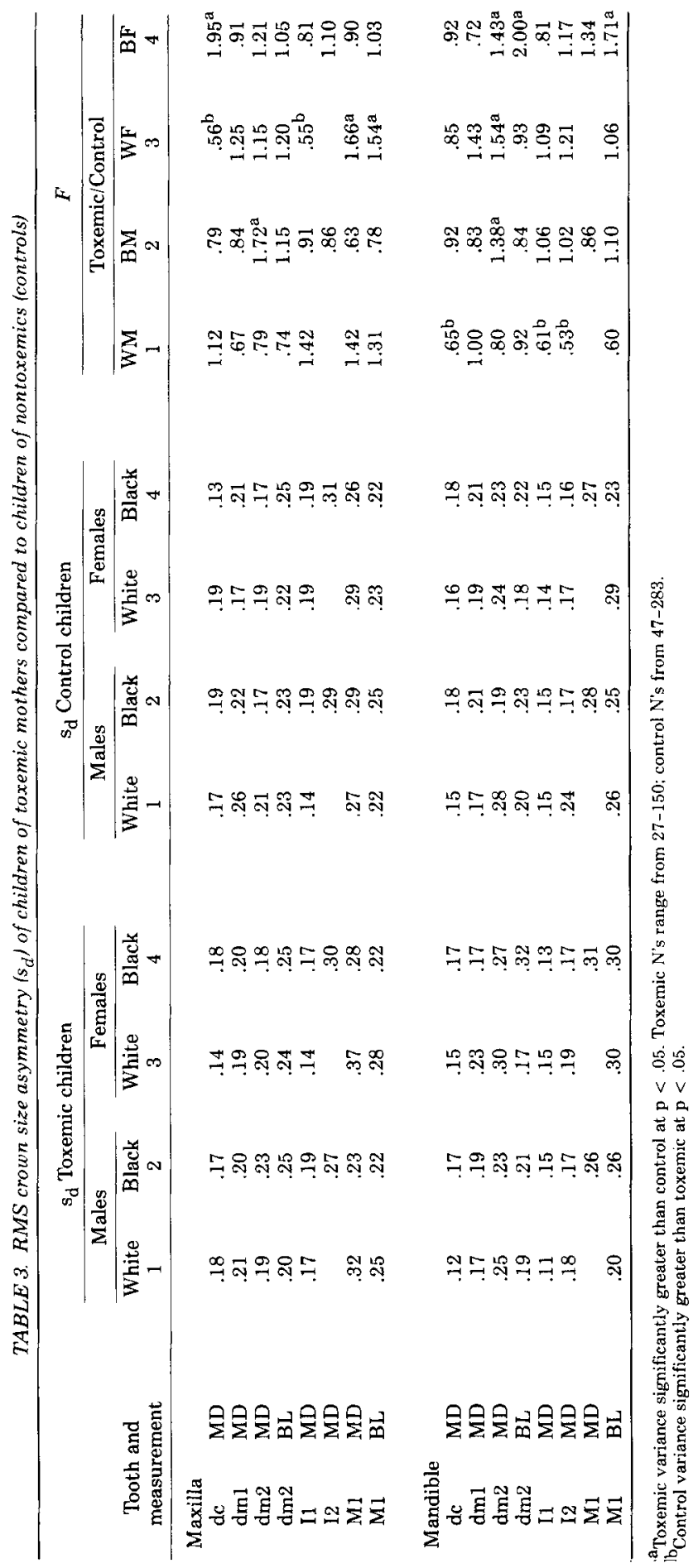


size (Garn et al., 1979), five comparisons out of 60 would support this hypothesis at $p<0.05$.

This comparison suggests two possibilities: (1) there is no increase in fluctuating dental asymmetry in children with these defined stressful prenatal and birth conditions, or (2) the true difference is small and we have made a Type II error due to a lack of sufficient statistical power. Although these two possibilities may seem to be singularly unhelpful (i.e., either we are right or wrong), the positive note is that more confidence in results could be achieved with larger sample sizes. Suppose that "stress" causes an increase in variance of left minus right tooth size of only $25 \%-$ a biologically and theoretically significant difference. If so, we will probably not find statistically significant results without two or three times sample sizes currently available (for example, if the true $F=1.25$, the tests in Table 3 had a power of only $15-40 \%$ ).

\section{CONCLUSIONS}

The results of these computer simulation, empirical, and mathematical studies are easily summarized. First, consideration should be given to the statistic employed to measure fluctuating dental asymmetry. Although for most purposes it may be desirable to correct for crown size differences $(r$ or 1-r), the absolute value of typical asymmetry (RMS asymmetry) may be more useful in other situations. Second, sample size is a major determinant of apparent crown size asymmetry, especially with samples of less than 100, due both to the small magnitude of left-right difference in crown size and small population differences in asymmetry. Most published crown size asymmetry values fall within the limits expected when sampling from a single population. The relationship between sample size and statistical power dictates that small differences in left minus right tooth size variance will not be detected unless studies increase sample sizes considerably. Indeed, with the largest currently available samples of children with defined stressful prenatal and birth conditions, we are still unable to support the hypothesis that prenatal "stress" increases fluctuating dental asymmetry.

In light of these findings, with both sampling limitations and the current inability to link increased levels of human dental asymmetry to any defined stress, we suggest that fluctuating dental asymmetry has not yet been established as a reliable measure of generalized "stress" in human populations. This is not to say that dental asymmetry cannot be studied or is without interest. Certainly it would be important to confirm a $25 \%$ increase in fluctuating dental asymmetry with developmental stress - although this finding would be of little practical importance for small archaeological samples. In this regard, experimental studies using high levels of a defined stress, such as those by Siegel and associates (Siegel et al., $1973,1975,1977)$, may be more rewarding. Human developmental stress and asymmetry might be better studied in congenital malformation syndromes in which the magnitude of the effect is large (Adams and Niswander, 1967; Garn et al., 1970; Barden, 1980; Sofaer, 1979; Wilcox, 1981) or in extreme prenatal stresses (such as the fetal alcohol syndrome).

In addition, there are structured aspects of dental asymmetry that merit study. Asymmetry appears to follow the predictions of gen eralized dental "field theory," with the more distal teeth in a class exhibiting more asymmetry (Moorrees and Reed, 1964; Garn et al., 1966). Similarly, the degree of asymmetry in maxillary and mandibular isomeres corresponds to a remarkable degree, whether measured as RMS asymmetry or as 1-r (Garn et al. 1981). The tendency for children with third molar agenesis to show increased asymmetry of other teeth (Garn et al., 1966) also deserves further attention in comparisons of different human populations since M3 agenesis is population specific.

Clearly, the factors that act to increase dental asymmetry are not yet understood, with the exception of chromosomal abnormalities and some single gene substitutions. Sample size alone has been a limiting factor in most studies to date. Ascertainment of the effect of prenatal and perinatal stresses on crown size asymmetry requires larger sample sizes than has been the custom and it will be useful to select defined stresses of known teratogenicity. Only then will we know whether we can reconstruct levels of stress for archaeological groups, again remembering the need for large samples. Dental asymmetry does exist, but more research is necessary to establish the meaning of both fluctuating and structured asymmetry in the dentition.

\section{ACKNOWLEDGMENTS}

We thank Dr. Richard H. Osborne for making the NCPP dental measurements available to us. This project was supported in part by contract N01-NS-2-2302 from the National Institute of Neurological Disorders and Stroke to 
the University of Wisconsin and contract N01-NS-5-2308 to the University of Michigan. Additional support came from grants $\mathrm{DE}$ 03443 and DE 03610 from the National Institute of Dental Research to the University of Michigan.

\section{LITERATURE CITED}

Adams, MS, and Niswander, JD (1967) Developmental "noise" and a congenital malformation. Genet. Res. Camb. 10:313-317.

Bader, PS (1965) Fluctuating asymmetry in the dentition of the house mouse. Growth 29:291-300.

Bailit, HL (1966) Tooth size variability, inbreeding, and evolution. Ann. N.Y. Acad. Sci. 134:616-623.

Bailit, HL, Workman, PL, Niswander, JD, and MacLean, CJ (1970) Dental asymmetry as an indicator of genetic and environmental conditions in human populations. Hum. Biol. 42:626-638.

Barden, HS (1980) Fluctuating dental asymmetry: A measure of developmental instability in Down syndrome. Am. J. Phys. Anthropol. 52:169-173.

Black, TK (1980) An exception to the apparent relationship between stress and fluctuating dental asymmetry. J. Dent. Res. 59:1168-1169.

Corruccini, RS and Potter, RHY (1981) Developmental correlates of crown component asymmetry and occlusal discrepancy. Am. J. Phys. Anthropol. 55:21-31.

DiBennardo, R, and Bailit, HL (1978) Stress and dental asymmetry in a population of Japanese children. Am. J. Phys. Anthropol. 48:89-94.

Dixon, WJ, and Massey, FJ (1969) Introduction to Statistical Analysis. New York: McGraw-Hill.

Doyle, WJ, and Johnston, $O$ (1977) On the meaning of increased fluctuating dental asymmetry: A cross popula. tion study. Am. J. Phys. Anthropol, 46:127-134.

Garn, SM, Cohen, MM, and Geciauskas, MA ( 1970) Increased crown-size asymmetry in Trisomy G. J. Dent. Res. 49:465.

Garn, SM, Lewis, AB, and Kerewsky, RS (1966) The meaning of bilateral asymmetry in the permanent dentition. Angle Ortho. 36:55-62

Garn, SM, Osborne, RH, and McCabe, KD (1979) The effect of prenatal factors on crown dimensions. Am. J. Phys. Anthropol. 51:655-677.
Garn, SM, Smith, BH, and Moyers, RE (1981) Structured (patterned) dimensional and developmental dental asymmetry. Proc. Finn. Dent. Soc. 77:33-36.

Moorrees, CFA, and Reed, RB (1964) Correlations among crown diameters of human teeth. Arch. Oral Biol. 9:685-697.

Moyers, RE, van der Linden, FPGM, Riolo, ML, and McNamara, JA (1976) Standards of Human Occlusal Development. Ann Arbor: The Center for Human Growth and Development. The University of Michigan.

Niswander, JD, and Chung, CS (1965) The effects of in breeding on tooth size in Japanese children. Am. J. Hum Genet. 17:390-398

Perzigian, AJ (1977) Fluctuating dental asymmetry: Variation among skeletal populations. Am. J. Phys. Anthropol. 47:81-88.

Potter, RH, and Nance, WE (1976) A twin study of dental dimension. I. Discordance, asymmetry, and mirror imagery. Am. J. Phys. Anthropol. 44:391-396.

Sciulli, PW, Doyle, WJ, Kelley, C, Siegel, P, and Siegel, MI (1979) The interaction of stressors in the induction of in creased levels of fluctuating asymmetry in the laboratory rat. Am. J. Phys. Anthropol. 50:279-284.

Siegel. MI, and Doyle, WJ (1975) The differential effects of prenatal and postnatal audiogenic stress on fluctuating dental asymmetry. J. Exp. Zool. 191:211-214.

Siegel, MI, Doyle, WJ, and Kelley, C(1977) Heat stress, fluc tuating asymmetry and prenatal selection in the laboratory rat. Am. J. Phys. Anthropol. 46:121-126.

Siegel, MI, and Smookler, HH (1973) Fluctuating dental asymmetry and audiogenic stress. Growth $37: 35-39$.

Sofaer, JA (1979) Human tooth-size asymmetry in cleft lip with or without cleft palate. Arch. Oral Biol. 24:141-146.

Staley, RN, and Green, LJ (1974) Types of tooth cusp occurrence asymmetry in human monozygotic and dizygotic twins. Am. J. Phys. Anthropol. 40:187-196.

Suarez, BK (1974) Neanderthal dental asymmetry and the probable mutation effect. Am. J. Phys. Anthropol. 41:411-416.

Van Valen, L (1962) A study of fluctuating asymmetry. Evolution 16:125-142.

Wilcox, J (1981) Bilateral Asymmetry of Tooth Size. Unpublished masters thesis, University of Toronto.

Wolpoff, MH ( 1975) Dental reduction and the probable mutation effect. Am. J. Phys. Anthropol. 43:307-308. 